\title{
Germanium Thermistor
}

\author{
Sergiy P. Pavlyuk, Valerii I. Grygoruk, Larisa V. Ishchuk and Olena S. Oberemok \\ Taras Shevchenko National University of Kyiv, 64/13, Volodymyrska Street, City of Kyiv 01601, Ukraine
}

\begin{abstract}
The main disadvantage of known thermistors is the weak sensitivity to a magnetic field what does not allow to control appreciably the resistance of a thermistor with the help of magnetic field. In addition, such a control is possible only at low temperatures. The new in the claimed technical solution is that the electrical contacts for a thermosensitive element are made of tin, and these contacts are able to inject nonequilibrium charge carriers into a thermosensitive element. The injection coefficient of contacts depends on the value of magnetic field which leads to an increase in the magnetic susceptibility of the thermistor. The new also is the production of a thermosensitive element from single-crystal germanium with the concentration of impurities less than thermodynamically equilibrium concentration of charge carriers at the temperature defined as "cold resistance" of a thermistor, i.e., the conduction is close to the intrinsic conduction at the above-mentioned temperature.
\end{abstract}

Key words: Exclusion, depletion, magnetic field, sensitivity.

\section{Introduction}

Semiconductor resistors [1-4] are well known for a long time and widely used in various devices. Their special feature is the dependence of the resistance on a magnetic field. This property enables to control the resistance of a thermistor by means other than cooling or heating, namely: by a magnetic field that widens the application of thermistors and allows using them in a new way. Electrical oxide semiconductor resistors described in [3], and other thermistors made from doped indium antimonide in which the predetermined value of the resistance is established by a magnetic field [4] are also well known.

Modern thermistors have a filament-like sensor with nearly rectangular cross-section, and they are made from single crystal germanium doped with gallium and zinc. Electrical contacts are ohmic and manufactured by using indium alloy. Disadvantages of these thermistors are low sensitivity to a magnetic field (the resistance in $10 \mathrm{kV}$ field varies less than 5\% at room temperature [5]) that allows to control the resistance of thermistors by a magnetic field only in a

Corresponding author: Sergiy P. Pavlyuk, Ph.D., senior researcher, research field: semiconductor physics. E-mail: pps@mail.univ.kiev.ua. narrow range, and the manufacturing process of ohmic contacts is complicated enough.

The aim of our study is to improve the magnetic sensitivity of the germanium thermistor and to simplify its manufacturing process.

The proposed germanium thermistor includes a thermosensitive element, electrical contacts and metal terminals. The thermistor can be mounted on a radiator. Let's consider the effect of constituent elements of the thermistor on its operation.

Electrical contacts for a thermosensitive element are made of tin, so they are able to inject unbalanced charge carriers into the element. The injection coefficient of such contacts depends on the magnetic field magnitude that improves the magnetic sensitivity of a thermistor.

The thermosensitive element is made from single-crystal germanium with the impurity concentration lesser than thermodynamic balanced concentration of charge carriers at the temperature defined as "cold resistance" of a thermistor. In this case the conduction is close to the intrinsic conduction at the above-mentioned temperature.

The "cold resistance" of a thermistor is the amount of its resistance at a certain temperature of environment [5]. For the proposed thermistor the 
"cold resistance" temperature is equal to $25{ }^{\circ} \mathrm{C}$. This allows us, when placing the thermistor in a magnetic field and reducing the injection efficiency of contacts, to achieve the carrier depletion for a thermosensitive element to the concentration lower than thermodynamic equilibrium. In this case, the bulk charge does not occur in it, and takes place the transition from the charge-carrier injection into the thermosensitive element (when a magnetic field is absent) to the charge-carriers exclusion from the element (in a magnetic field) which improves the magnetic sensitivity of the thermistor.

In germanium thermistors that are described in Refs. [1-4] thermosensitive elements have been made of doped or compensated single-crystal germanium, and electrical contacts are ohmic. The variation range of the injection coefficient of these ohmic contacts, under the influence of a magnetic field, is less than the variation range of the injection coefficient of tin contacts used in this study, since the latters are able to inject nonequilibrium charge carriers into a thermosensitive element. As a result, the thermistor with ohmic contacts has a lower magnetic sensitivity compared to the proposed thermistor. If the contacts are made by burning or fusing them into, their injection coefficient does not depend practically on the value of magnetic field [6] which lowers the magnetic sensitivity as well.

Furthermore, the manufacturing of a thermosensitive element from doped or compensated germanium does not allow depleting charge carriers of a thermosensitive element to the concentration lower than the impurity concentration in it, without the occurrence of bulk charge.

As a result the exclusion effect is impossible, and that also leads to the lower sensitivity of known thermistors compared to the proposed one. The proposed thermistor has a thermosensitive element shaped as a parallelepiped with dimensions $0.135 \times$ $0.06 \times 0.625 \mathrm{~cm}^{3}$, made of monocrystalline germanium with the conduction close to his own: the donor concentration is $3.4 \times 10^{11} \mathrm{sm}^{-3}$ and the lifetime of charge carriers is $10^{-4} \mathrm{sec}$. Electrical contacts are made by the soldering of chemically pure tin onto the element's tip.

\section{Experimental Results}

Fig. 1 shows the CVC (current-voltage characteristics) of the proposed thermistor at the temperature $\mathrm{T}=25{ }^{\circ} \mathrm{C}$ in a magnetic field with varying intensity. From the curves in Fig. 1 (the curve 1 corresponds to CVC of the thermistor without a magnetic field, the curves $2,3,4,5$ correspond to magnetic fields with intensity $\mathrm{H}=1 \mathrm{kOe}, 3 \mathrm{kOe}, 6$ $\mathrm{kOe}, 9 \mathrm{kOe}$ ) we can see that the ratio of resistances of the thermistor at CVC peaks in a magnetic field with $\mathrm{H}=9 \mathrm{kOe} \mathrm{R} 9$ (curve 5) and without a magnetic field $\mathrm{R} 0$ (curve I) R9/R0 = 10. In other words, the relative change in resistance R9-R0/R0 of the proposed thermistor in the $9 \mathrm{kOe}$ field reaches $900 \%$ what is 180 times higher than the sensitivity to a magnetic field of existing thermistors [1-4].

\section{Results and Discussion}

The possibility to obtain the high sensitivity to a

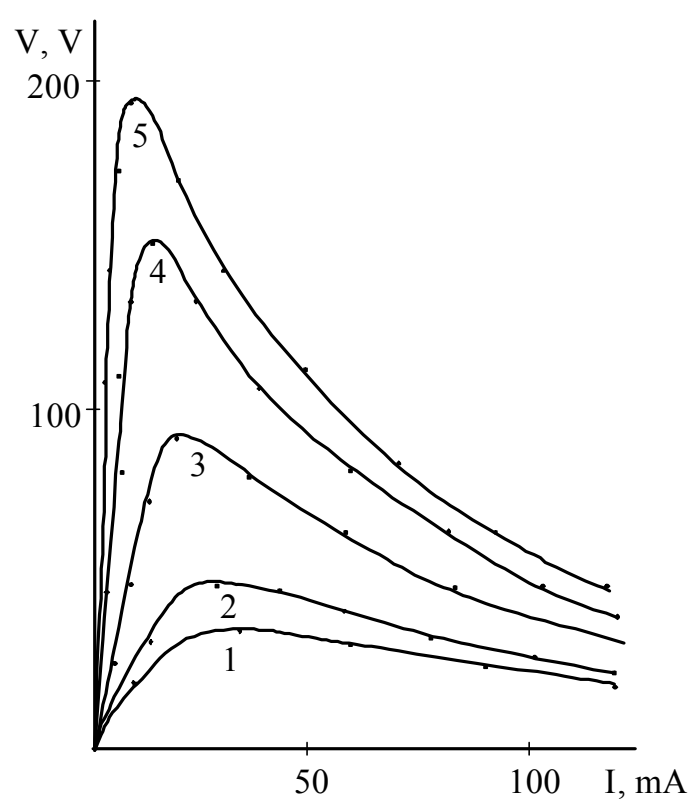

Fig. 1 Current-voltage characteristics of the proposed thermistor at the temperature $\mathrm{T}=25^{\circ} \mathrm{C}$ in a magnetic field with varying intensity. 
magnetic field for the proposed thermistor, and hence to control its CVC over a wide range with the help of a magnetic field is based on the change in injection coefficient of contacts by a magnetic field and on the appearance of charge carrier exclusion from the thermosensitive element [6].

The proposed thermistor works in the following way: when current flows through it we can observe typical CVC for thermistors with negative temperature coefficient of the resistance, which comprises two main sections and has a maximum voltage. At first, with the increase of the current passing through the thermistor the voltage increases too (the first section). Then, with the heating-up of the thermosensitive element by the current flowing through it, its resistance decreases, and the voltage on the thermistor falls. We can observe a falling part of CVC (the second section). The reducing of the resistance of the thermosensitive element is caused by the increase in a number of charge carriers in it as a result of the thermal supplying of electrons in the conduction band and the appearance of an equal number of holes to electrons in the valence band.

Tin contacts inject unbalanced charge carriers into a thermosensitive element that makes the first section of $\mathrm{CVC}$ be more inclined than in the thermistor with ohmic contacts, which changes the position of its maximum, shifting it in the direction of lower voltages.

When we place our thermistor in a magnetic field the injection coefficient of contacts decreases, and accordingly, the amount of injected unbalanced charge carriers decreases too. The reducing of the injection level changes CVC: as the amount of injected charge carriers decreases, the first section becomes steeper, and CVC maximum is shifted to the region of lower currents and high voltages. At that, the resistance of the thermistor at CVC maximum increases with the increase of magnetic field $\mathrm{H}$. In addition, the steepness of falling section also increases [7].

As a magnetic field increases, one can observe the decrease of the injection of unbalanced charge carriers into the thermosensitive element and the turn to the charge carrier exclusion from the thermosensitive element. The effect of the exclusion increases the resistance in a magnetic field and then enhances the magnetic sensitivity of our thermistor. In the magnetic field of the thermistor, in addition to the decrease of injection coefficient of contacts, many processes changing the resistance have been observed: the effects of magnetoresistance and magnetoconcentration. However, in comparison with them the process of reducing the injection coefficient in contacts is determinant [6].

When we place the thermistor on a radiator its magnetosensitivity increases. This may be explained in the following way. The heatsink of a radiator increases the dispersion coefficient value [1]; as a result CVC maximum is shifted to higher currents and voltages compared with CVC maximum of the thermistor without a radiator. High voltages applied to the thermistor, when it is placed on a radiator, allow realizing in a magnetic field the stronger exclusion of charge carriers from the thermosensitive element than in the case of the thermistor without a radiator, and this is equivalent to an increase in the magnetic sensitivity.

\section{Conclusion}

The proposed thermistor has the following advantages compared to the existing thermistors.

(1) Higher sensitivity to a magnetic field: the sensitivity of the proposed thermistor without a radiator is 180 times higher than the sensitivity of existing thermistors, and with a radiator- 580 times higher.

(2) Easy manufacturing technology: the proposed thermistor has no need for ohmic contacts.

(3) The opportunity to create a variety of regulated and controlled by magnetic field devices, such as multivibrators, relays, variable resistors without a sliding contact, and magnetic field sensors. 


\section{References}

[1] Sheftel, I. T. 1973. Thermoresistors. Moscow: Nauka. (in Russian)

[2] Meklin, E. 1983. Thermoresistors. Moscow: Radiosviaz. (in Russian)

[3] Patent of Federal Republic of Germany. 1951. ЖЮ8851, НКИ 21с-54/O5.

[4] Patent of Federal Republic of Germany. 1973. И490498 МКИ Н01с 7/O4.

[5] Vasilevskaya, V. N., Vinetskiy, R. M., Miselyuk, E. G.,
Poletayeva, I. F., and Fortunatova, N. N. 1967. "Germanium Thermoconverter of the Resistance for Temperatures 20-77K." Poluprovodnicovaya Technica i Microelectronica (Semiconductor Techniques and Microelectronics) 2: 13-22. (in Russian)

[6] Zeeger, K. 1977. Fizica poluprovodnicov (Physics of Semiconductors). Moscow: Mir. (in Russian)

[7] Dobrovolskiy, V. N., Vorobyiva, D. L., and Pavlyuk, S. P. 1985. "Reducing of Injection and Exclusion Stimulated by Magnetic Field." Physics and Techniques of Semiconductors 19 (4): 687-91. (in Russian) 